\title{
CZT drift strip detectors for high energy astrophysics
}

\author{
Kuvvetli, Irfan; Budtz-Jørgensen, Carl; Caroli, E.; Auricchio, N.
}

Published in:

Nuclear Instruments and Methods in Physics Research Section A: Accelerators, Spectrometers, Detectors and Associated Equipment

Link to article, DOI:

10.1016/j.nima.2010.03.172

Publication date:

2010

Link back to DTU Orbit

Citation (APA):

Kuvvetli, I., Budtz-Jørgensen, C., Caroli, E., \& Auricchio, N. (2010). CZT drift strip detectors for high energy astrophysics. Nuclear Instruments and Methods in Physics Research Section A: Accelerators, Spectrometers, Detectors and Associated Equipment, 624(2), 486-491. https://doi.org/10.1016/j.nima.2010.03.172

\section{General rights}

Copyright and moral rights for the publications made accessible in the public portal are retained by the authors and/or other copyright owners and it is a condition of accessing publications that users recognise and abide by the legal requirements associated with these rights.

- Users may download and print one copy of any publication from the public portal for the purpose of private study or research.

- You may not further distribute the material or use it for any profit-making activity or commercial gain

- You may freely distribute the URL identifying the publication in the public portal

If you believe that this document breaches copyright please contact us providing details, and we will remove access to the work immediately and investigate your claim 
Provided for non-commercial research and education use. Not for reproduction, distribution or commercial use.

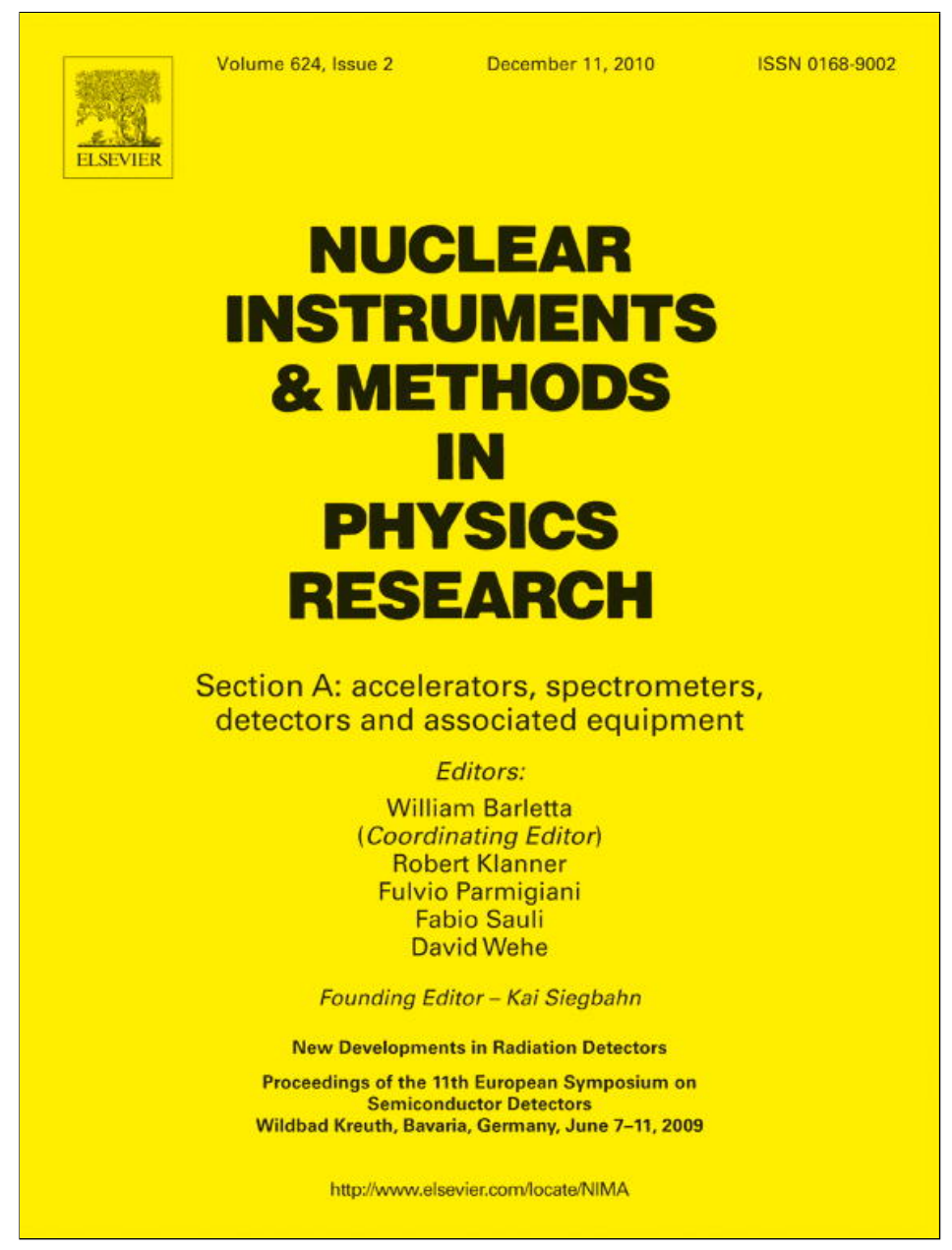

This article appeared in a journal published by Elsevier. The attached copy is furnished to the author for internal non-commercial research and education use, including for instruction at the authors institution and sharing with colleagues.

Other uses, including reproduction and distribution, or selling or licensing copies, or posting to personal, institutional or third party websites are prohibited.

In most cases authors are permitted to post their version of the article (e.g. in Word or Tex form) to their personal website or institutional repository. Authors requiring further information regarding Elsevier's archiving and manuscript policies are encouraged to visit:

http://www.elsevier.com/copyright 


\title{
CZT drift strip detectors for high energy astrophysics
}

\author{
I. Kuvvetli ${ }^{\text {a,*}}{ }^{2}$ C. Budtz-Jørgensen ${ }^{\text {a }}$, E. Caroli ${ }^{\text {b }}$, N. Auricchio ${ }^{c}$ \\ a DTU Space, National Space Institute, Denmark \\ ${ }^{\mathrm{b}}$ INAF-IASF, Univ. of Bologna, Italy

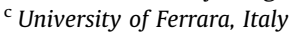

\section{A R T I C L E I N F O}

\section{Available online 10 April 2010}

\section{Keywords:}

Compound semiconductors

$\mathrm{X}$-ray detection

Gamma-ray detection

Depth sensing

CZT drift strip detectors

High energy astrophysics instrumentation

\begin{abstract}
A B S T R A C T
Requirements for X- and gamma ray detectors for future High Energy Astrophysics missions include high detection efficiency and good energy resolution as well as fine position sensitivity even in three dimensions.

We report on experimental investigations on the CZT drift detector developed DTU Space. It is operated in the planar transverse field (PTF) mode, with the purpose of demonstrating that the good energy resolution of the CZT drift detector can be combined with the high efficiency of the PTF configuration. Furthermore, we demonstrated and characterized the 3D sensing capabilities of this detector configuration.

The CZT drift strip detector $(10 \mathrm{~mm} \times 10 \mathrm{~mm} \times 2.5 \mathrm{~mm})$ was characterized in both standard illumination geometry, Photon Parallel Field (PPF) configuration and in PTF configuration. The detection efficiency and energy resolution are compared for both configurations . The PTF configuration provided a higher efficiency in agreement with calculations. The detector energy resolution was found to be the same (3 keV FWHM at $122 \mathrm{keV}$ ) in both in PPF and PTF .

The depth sensing capabilities offered by drift strip detectors was investigated by illuminating the detector using a collimated photon beam of ${ }^{57} \mathrm{Co}$ radiation in PTF configuration. The width $(300 \mu \mathrm{m}$ FWHM at $122 \mathrm{keV}$ ) of the measured depth distributions was almost equal to the finite beam size. However, the data indicate that the best achievable depth resolution for the CZT drift detector is $90 \mu \mathrm{m}$ FWHM at $122 \mathrm{keV}$ and that it is determined by the electronic noise from the setup.
\end{abstract}

(c) 2010 Elsevier B.V. All rights reserved.

\section{Introduction}

The upcoming X-ray astrophysics missions in the $\mathrm{keV}$ to $\mathrm{MeV}$ band require instrumentation advanced in both spectral and imaging capabilities of the detectors and in the capabilities of X-ray imaging optics. The instrumentation for these types of telescopes requires detectors of high efficiency, with energy resolution on the order of a few $\mathrm{keV}$ and the capability of three dimensional (3D) position sensitivity.

Compound room temperature semiconductor detectors such as CdZnTe (CZT) and CdTe are good candidates for hard X-ray ( $>10 \mathrm{keV}$ ) and $\gamma$-ray astronomy instrumentation. A major drawback for these type of detectors is the ineffective charge collection within the detector, especially for the holes which affect and degrade the detectors' spectral performance. At DTU Space, the development of CZT Drift Strip detectors [1] and [2], resulted in significant spectral performance improvements.

\footnotetext{
* Corresponding author.

E-mail address: irfan@space.dtu.dk (I. Kuvvetli).
}

\section{CZT drift strip detector}

Fig. 1 shows the principle of CZT drift strip detectors. The detector structure is similar to the Silicon drift detector which was first time introduced by Emilio Gatti and Pavel Rehak in 1983 [3]. A CZT drift strip detector cell is shown between the dashed lines marked with A and B. The structure employs a number of drift strips (small black boxes) separating the anode readout strips (small white boxes labeled as $Q_{s}$ ) on one side and a planar electrode on the other. A voltage divider supplies each drift strip with a bias of $V_{i}=V_{d} \times(i / 4),(i=1,2,3,4)$, while the anode strips are held at ground potential. The detector is biased such that the electrons, produced by the photon interaction, are drifted to an anode readout strip with their transport properties (mobilitylifetime product, $\mu \tau_{e}$, up to $10^{-2} \mathrm{~cm}^{2} / \mathrm{V}$ for CZT). The positive charges (holes) produced by the photon interaction have a poor mobility-lifetime product $\left(\mu \tau_{h}\right.$, values up to $\left.10^{-5} \mathrm{~cm}^{2} / \mathrm{V}\right)$ in CZT and will, with high probability, be trapped in the detector. However, the anode signal is unaffected by the holes since the anode strips are screened by the bias strips [1]. The anode signals are therefore proportional to the photon energy and high spectroscopic performance is ensured for CZT materials with 


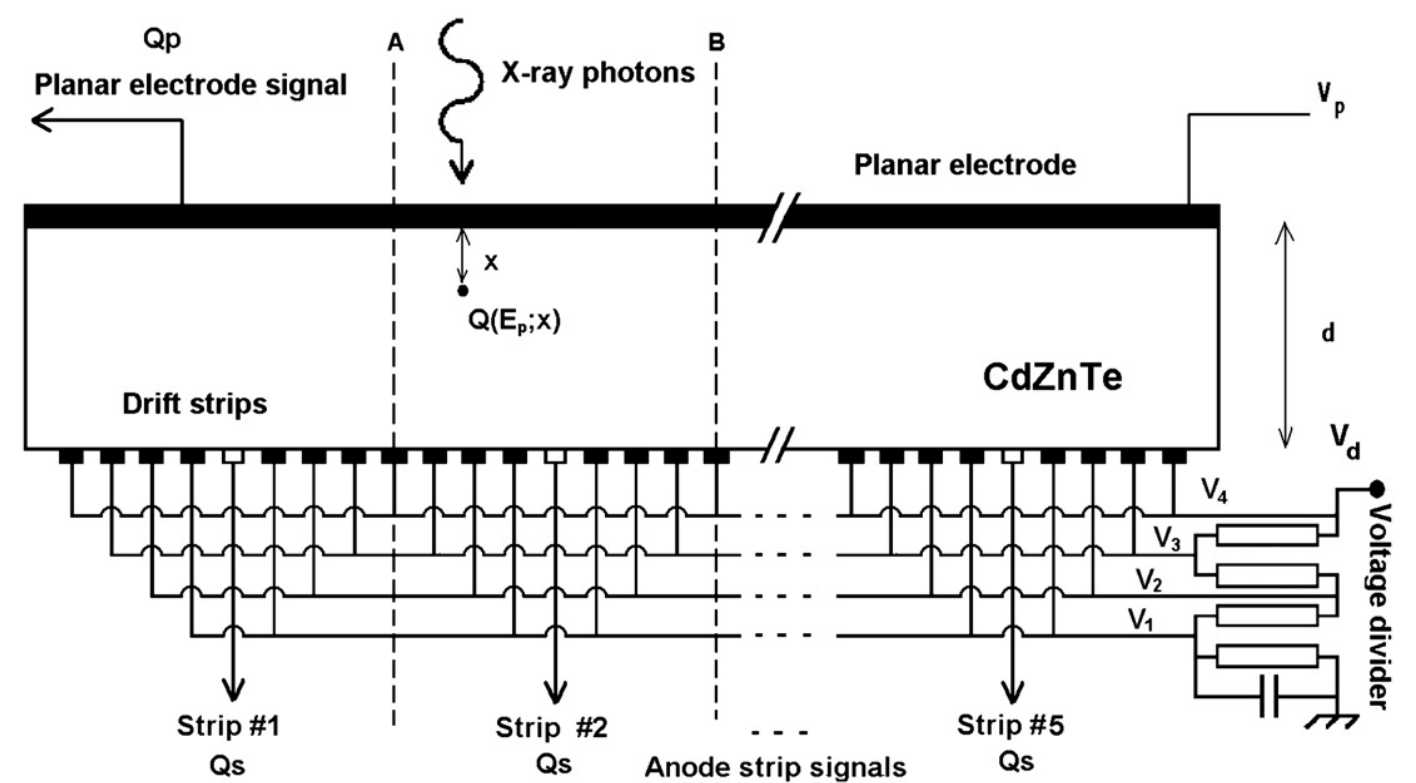

Fig. 1. Principle of the CZT drift strip detector. A drift strip detector cell is shown between dashed lines marked with "A" and "B". The drift strip electrodes and the planar electrode are biased in such a way that the electrons move to the anode strips (white boxes).

good electron drift properties. Although the spectroscopic properties of these detectors are almost independent of material hole transport properties, they are of course very dependent on the electron transport properties and especially, materials with fluctuating electron trapping lengths will result in degraded detector performance.

Not only does the drift strip readout technique provide an improved energy determination for CZT detectors, but it also yields information about the interaction depth of the detected photon. The depth information (depth sensing) can be derived from the ratio, $R \equiv Q_{p} / Q_{s}$, where $Q_{p}$ is the planar electrode signal and $Q_{s}$ the anode strip signal. The quantity $R$ is almost linearly dependent on the photon interaction depth, $x$, with a value close to unity for interactions close to the planar electrode and a value close to zero for interactions near the strip electrodes. For further details see Ref. [1]. The depth of interaction (DOI) information can be used to correct residual electron trapping effects on the anode signal and improve further the detectors energy resolution.

Some of the best spectral performance figures for drift strip detectors have been reported for full illumination over a wide energy range in Refs. [4-6]. It is demonstrated in these reports that the CZT drift strip detectors can achieve energy resolutions which are within a factor of 2 to 3 of the CdZnTe Fano-limited resolution.

\section{Applications}

Instruments based on CZT drift strip detector systems have been proposed for for a number of space missions:

(1) The X-Ray Imager (XRI) on the Atmospheric X-ray Observatory (AXO) [7]. The AXO mission was proposed by the Danish Small Satellite program and was dedicated to the observation of X-rays generated in the Earth's atmosphere. The AXO XRI was an imaging instrument using a coded mask and a 2D $800 \mathrm{~cm}^{2}$ CZT drift strip detector.

(2) AXO XRI was developed further and proposed as the Modular X-ray and Gamma-ray Sensor (MXGS) on board the Atmosphere Space Interactions Monitor (ASIM) [8]. ASIM is an accepted European Space Agency (ESA) mission for the
International Space Station. It will study giant electrical discharges (lightning) in the high-altitude atmosphere above thunderstorms. The discharges are seen as optical, $\mathrm{X}$-ray and Gamma-ray flashes in the stratosphere and the mesosphere.

(3) As focal plane detector for the Gamma-Ray Imager (GRI) [9] and [10]. The Gamma-Ray Imager was proposed for the ESA Cosmic Vision 2015-2025 plan. It was not accepted in the first round, but development work continues. GRI proposed for the first time the use of novel focusing optics to concentrate high energy photons on a small focal spot. The energy coverage of $10 \mathrm{keV}-1.3 \mathrm{MeV}$ will be achieved by combining a Laue crystal lens with a single-reflection multilayer-coated mirror. The GRI focal plan detector contains four stacked CZT layers operated in PTF configuration, surrounded by CZT side walls with about the same characteristics in terms of thickness and spatial resolution. The focal plane detector is based on 3D positionsensitive CZT drift strip detectors and was designed to optimize the response for the Point Spread Function characteristics foreseen for the GRI focusing optics.

\section{Detector illumination geometry}

Fig. 2 shows the possible illumination geometries used for CZT drift strip detectors. (a) Standard illumination geometry, Photon Parallel Field (PPF). The photons enter the detector perpendicular to the planar electrode. (b) Side illumination configuration, Photon Transverse Field (PTF). The photons enter the detector perpendicular to the side of the detector. This mode has the advantage that photons can be absorbed in the full length of the detector while the created charge at most will drift through the thickness of the detector. Therefore this detector illumination mode will provide high efficiency, preserving the excellent spectroscopic performance obtained for the CZT drift strip detectors. (c) PTF configuration as in (b) but with segmented planar electrodes orthogonal to the strips which achieves position sensitivity in the direction along the strips.

In this paper we report for the first time on results obtained for CZT drift strip detectors operated in PTF configuration as shown in (b). 


\section{Experimental setup}

The CZT drift strip detector was fabricated of spectroscopic grade material from eV Products, with a size of $10 \mathrm{~mm} \times 10 \mathrm{~mm}$ $\times 2.5 \mathrm{~mm}$. Electrodes deposited on the CZT detector material consisted of strips with a double layer of $\mathrm{Pt} / \mathrm{Au}$ electrodes and a planar cathode with a single layer of Pt electrode. The strip pitch was $200 \mu \mathrm{m}$ with a $100 \mu \mathrm{m}$ strip width. Each drift detector cell consists of $8 \mathrm{drift}$ strip electrodes ( 4 on each side of the anode readout strip) and one anode readout strip. The anode readout strips are held at ground potential and the drift strips are negatively biased by a voltage divider providing: $V i=V d \times i / 4$, $(i=1,2,3,4)$, where $V d$ is the drift bias. The detector was operated at $V p=-150 \mathrm{~V}$ and $V d$ follow: $\mathrm{V} 1=-30 \mathrm{~V}, \mathrm{~V} 2=-60 \mathrm{~V}$, $\mathrm{V} 3=-90 \mathrm{~V}, \mathrm{~V} 4=-120 \mathrm{~V}$. These measurement settings were used for all measurements in this paper.

Seven preamplifiers (eV-550) were used to read out 6 anode strips and one planar electrode. Each preamplifier output signal was connected to a main shaping amplifier and then fed to an ADC of the multi-parameter data acquisition system. For the spectral performance characterization work, data were recorded by illuminating the detector with photons from ${ }^{57} \mathrm{Co},{ }^{241} \mathrm{Am}{ }^{109} \mathrm{Cd}$ and ${ }^{137} \mathrm{Cs}$ sources in both PTF and PPF configurations.

For the depth sensing capabilities, a 2D scan using a collimated photon beam from a ${ }^{57}$ Co source (collimator hole $200 \mu \mathrm{m} \varnothing$ ) was

a

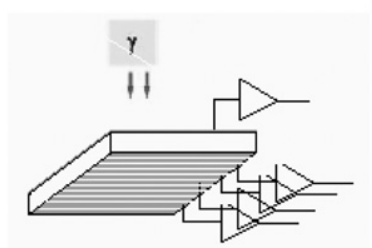

Fig. 2. Drift strip detector illumination geometries. (a) PPF configuration, (b) PTF configuration, (c) PTF configuration with segmented planar electrodes. conducted in PTF configuration. The PTF detector scan setup is shown in Fig. 3(a). The beam collimator was moved by steps of $0.1 \mathrm{~mm}$ in both directions, illuminating the detector on the side surface. The beam profile was measured for the scan setup using 1D scan of the edge of the detector, advancing the beam by steps of $0.01 \mathrm{~mm}$ from the planar side to the strip side.

The beam profile data are shown in Fig. 3(b). The derivative of the total counts registered by the planar electrode was used for the beam profiling. The sharp edge of the detector and the detector depletion zone, at the beam entry locations, close to the planar electrode, were investigated using the recorded spectra. The data showed that the detector was fully depleted and spectra at the edge locations, close to the planar electrode demonstrated excellent charge collection. The beam spot size on the detector surface was measured to be $230 \mu \mathrm{m}$ FWHM for a collimator height of $14 \mathrm{~cm}$.

\section{Experimental results}

\subsection{Spectral performance}

The detector energy resolution and efficiency for both PPF and PTF illumination configurations were compared using recorded detector data for full illumination with photons from ${ }^{57} \mathrm{Co},{ }^{241} \mathrm{Am}$, ${ }^{109} \mathrm{Cd}$ and ${ }^{137} \mathrm{Cs}$ sources. Fig. 4(a) shows the energy resolution as a function of energy, in both configurations. The measured energy spectra were not corrected using the DOI method. The results shown in Fig. 4 indicate that the CZT drift strip detector spectral performance for PTF and PPF is equal for the observed energy range.

Fig. 4(b) shows the normalized ${ }^{57} \mathrm{Co}$ spectra obtained by the detector (from strip2) using both PPF and PTF configurations. The detector has high efficiency due to a large effective thickness of $10 \mathrm{~mm}$ in PTF compared to the effective thickness of $2.5 \mathrm{~mm}$ in PPF. The energy resolution for the two spectra are equal. The PTF configuration demonstrates both high efficiency, due to larger effective thickness, without loosing detector's good energy resolution thanks to the unchanged small drift length for the electrons.

Fig. 5 shows the count ratio Nppf/Nptf (star points) as a function of energy. Nppf is the total number of counts under the photopeaks $(57.5,122,136 \mathrm{keV})$ for the PPF configuration and Nptf is the counts for the PTF for the same energies. The calculated a

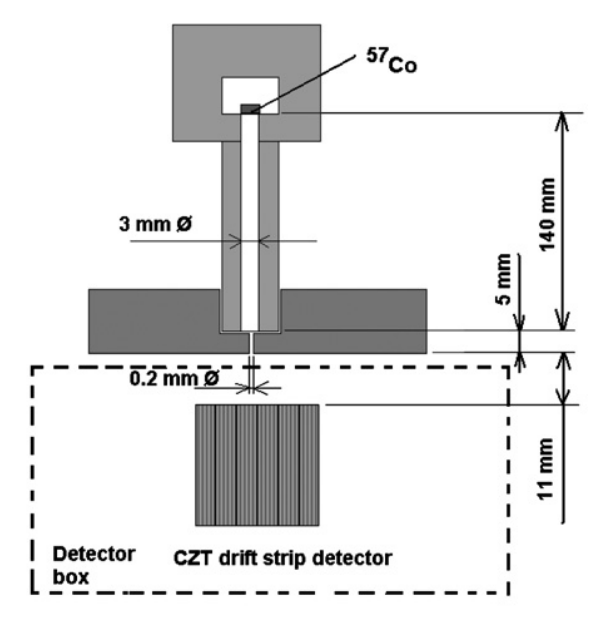

b

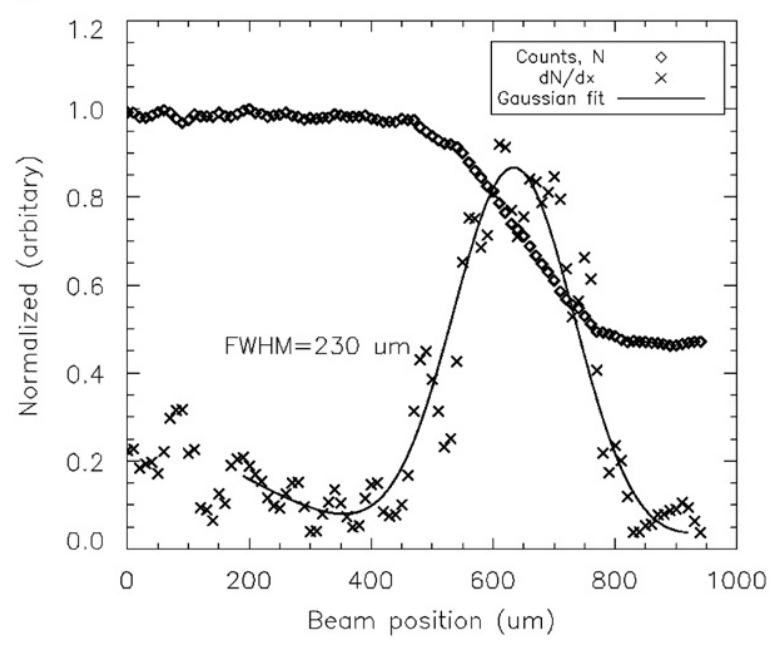

Fig. 3. $2 \mathrm{D}$ detector scan setup. (a) ${ }^{57}$ Co collimator setup (not to scale) and (b) photon beam profile. 
a

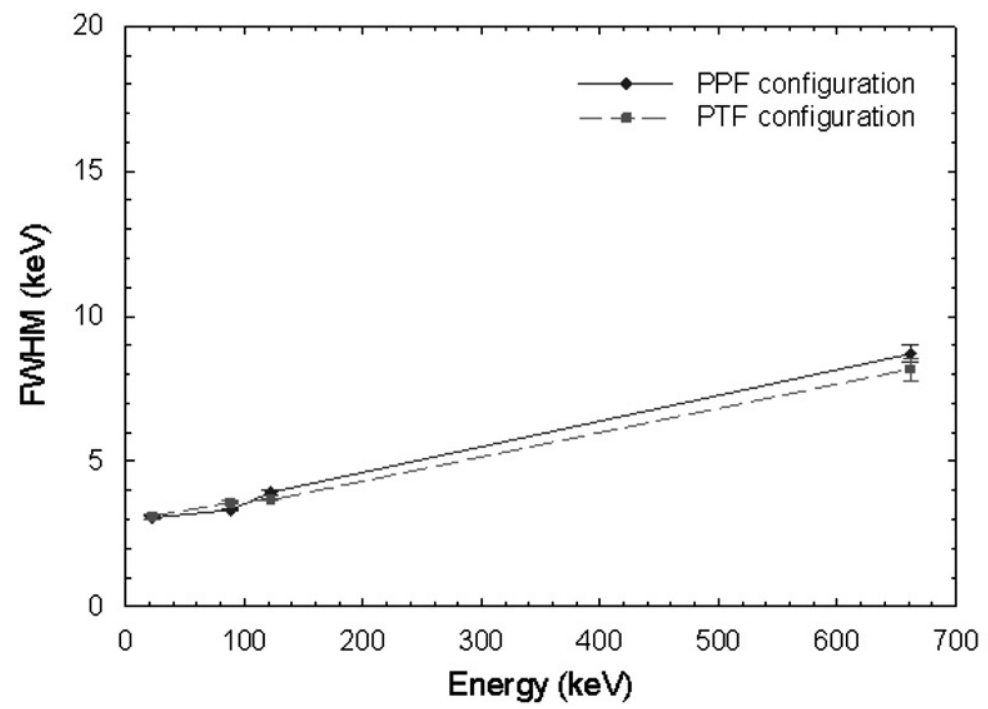

b

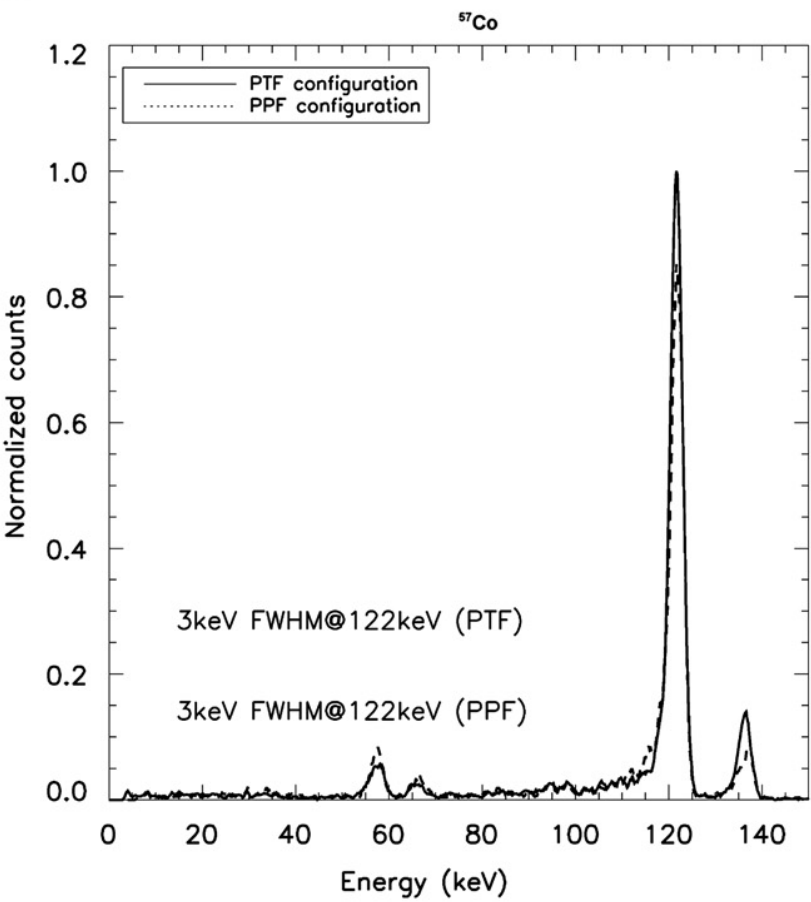

Fig. 4. PTF versus PPF spectral performance comparison. (a) Energy resolution as a function of energy (DOI correction is not applied). (b) Compression of normalized ${ }^{57}$ Co spectra obtained by PPF and PTF configurations.

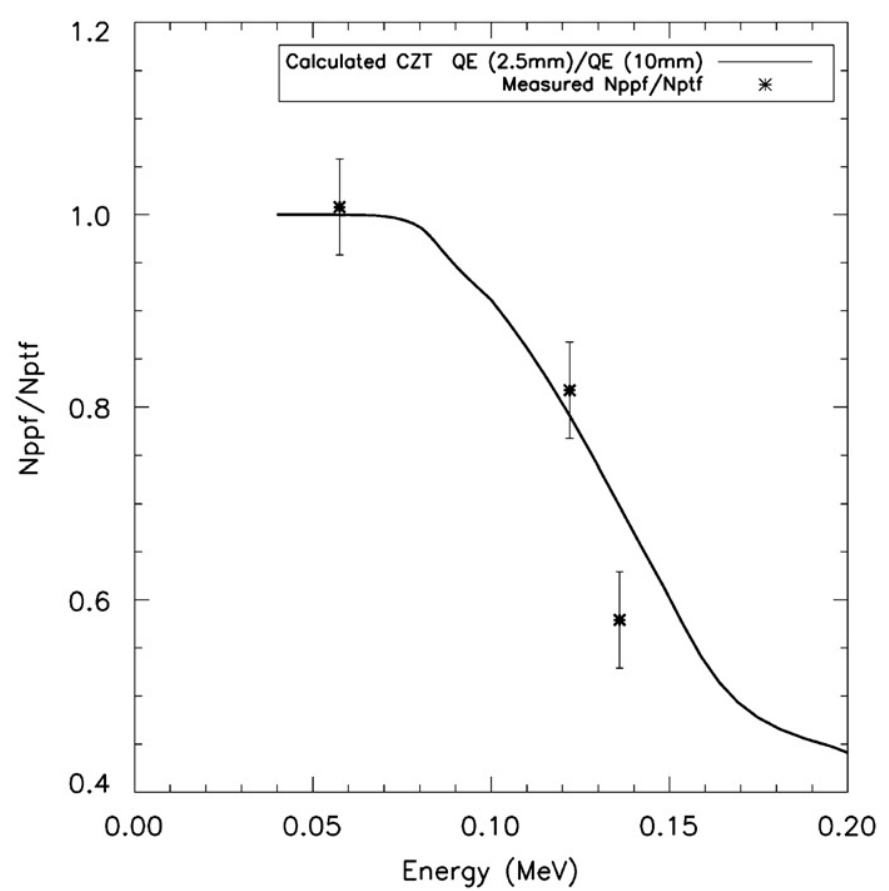

Fig. 5. The measured ratios Nppf/Nptf are shown as star points where Nppf and Nptf are the total counts under the photopeak for the PPF and the PTF configurations, respectively. The calculated ratio of quantum efficiencies for the PPF and the PTF as a function of energy is shown as solid line.

ratio of quantum efficiencies is shown (solid line) in the figure for 2.5 and $10 \mathrm{~mm}$ CZT as a function of energy. The result shows that the measured ratio $\mathrm{Nppf} / \mathrm{Nptf}$ and calculated $\mathrm{QE}(2.5 \mathrm{~mm}) /$ $\mathrm{QE}(10 \mathrm{~mm})$ values agree for the the observed energy range.

\subsection{Depth sensing}

The depth sensing capabilities of the CZT drift strip detector [2] were investigated with the present setup. The areas of two drift detector cells were scanned with a collimated ${ }^{57} \mathrm{Co}$ photon beam $(230 \mu \mathrm{m} \varnothing)$ using steps of $0.1 \mathrm{~mm}$ in both directions ( $x$ and $y$ ). Fig. 6(a) shows the detector geometry used for the scan and single line scan with the beam stepping from the planar electrode to the anode strip. Fig. 6(b) shows the ${ }^{57}$ Co data for the selected 3 beam positions. These three positions are representative of the 31 data sets. Fig. 6(b) left shows the bi-parametric distributions of the ratio, $R$ between the planar electrode signal, $Q_{p}$ and the strip signal, $Q_{s}$ versus $Q_{s}$. Fig. 6(b) right shows the three measured depth spectra. The derived $R$ distributions for the $122 \mathrm{keV}$ line were fitted and the FWHM values are given with each peak.

The width of the measured depth distribution corresponds to $300 \mu \mathrm{m}$ FWHM at $122 \mathrm{keV}$ and is mainly given by the finite beam size of $230 \mu \mathrm{m}$. The achievable depth resolution is determined by the electronic noise of the $Q_{p}$ and $Q_{s}$ signals. The achievable depth resolution as a function of the electronic noise and energy is given in Eq. (1). Here $Q_{s}$ is independent of material hole collection characteristics and therefore represents the full energy signal of the detected photon.

$R_{\text {noise }}=\frac{1}{Q_{s}} \times \sqrt{Q_{\text {pnoise }}^{2}+R^{2} \times Q_{\text {snoise }}^{2}}$

For the current detector scan setup, the electronic noise, $Q_{\text {pnoise, }}$ was $3.6 \mathrm{keV}$ FWHM for the planar signal $Q_{p}$, and $Q_{\text {snoise }}$ was $2.1 \mathrm{keV}$ FWHM for anode strip $Q_{s}$. Therefore, the achievable $R$ resolution is $90 \mu \mathrm{m}$ FWHM at $122 \mathrm{keV}$ according to Eq. (1).

Fig. 7(a) shows measured and derived $R$ values versus depth for energies of $57.5,122$ and $136 \mathrm{keV}$. The quantity $R$ is linearly dependent on the beam position $x$, with a value close to unity for interactions close to the planar electrode and a value close to zero for interaction near the strip electrodes. The minimum $R$ value 
a

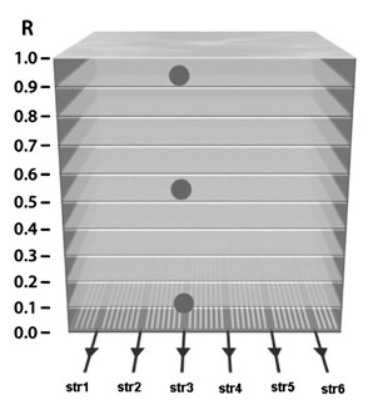

b

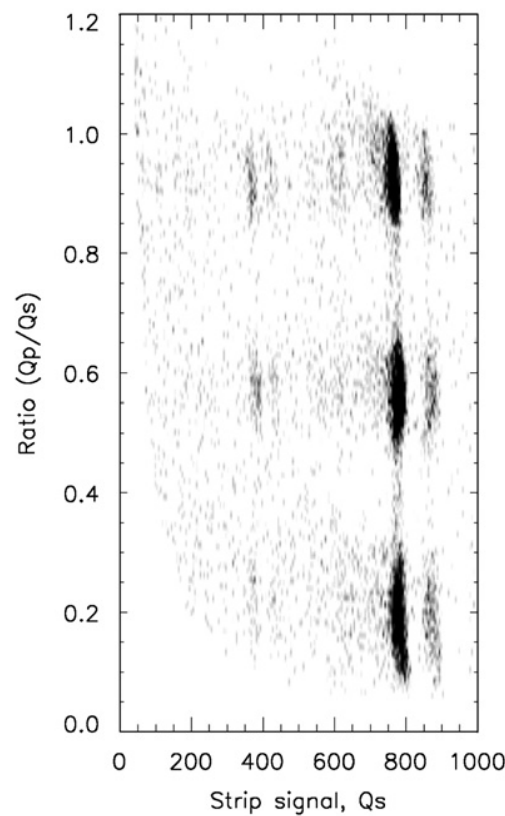

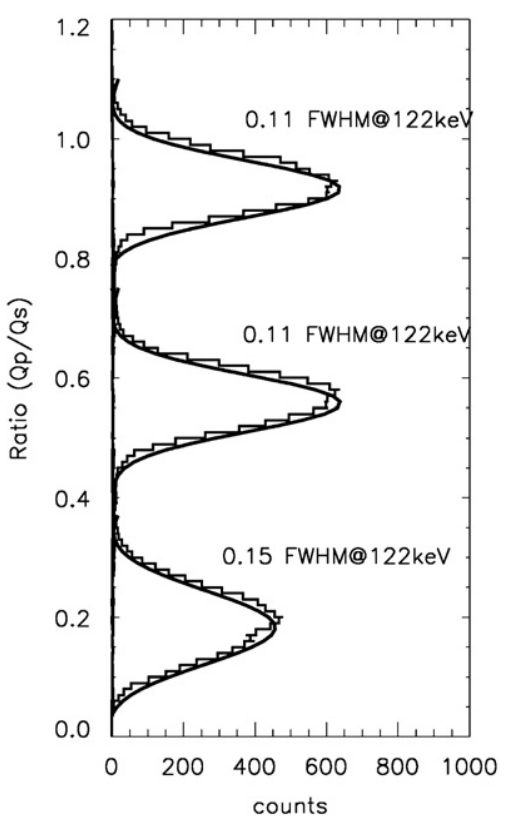

Fig. 6. (a) The detector geometry and beam scan orientation (not to scale), (b) left: the bi-parametric distributions of the ratio, between the planar electrode signal, Qp and the strip signal, Qs versus Qs for 3 different ${ }^{57}$ Co beam positions. (b) right: The corresponding measured depth resolution for these three different beam positions.

a

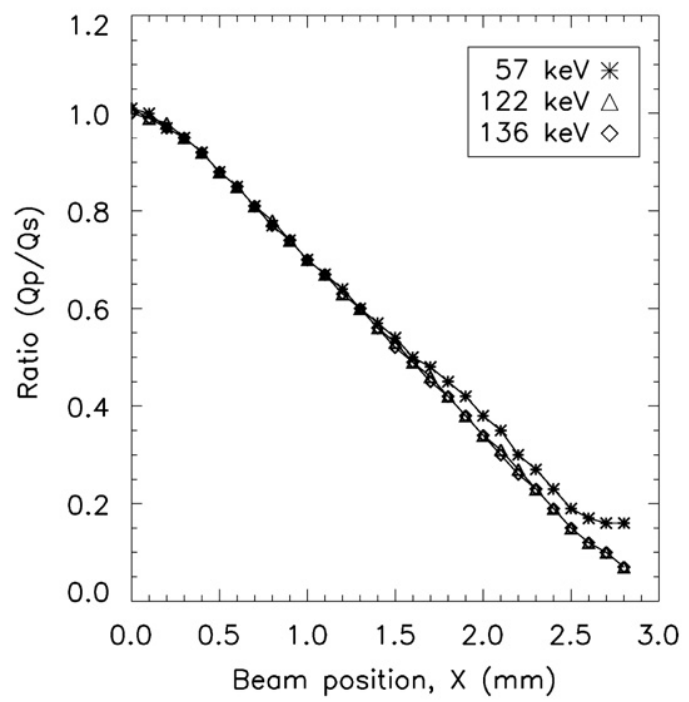

b

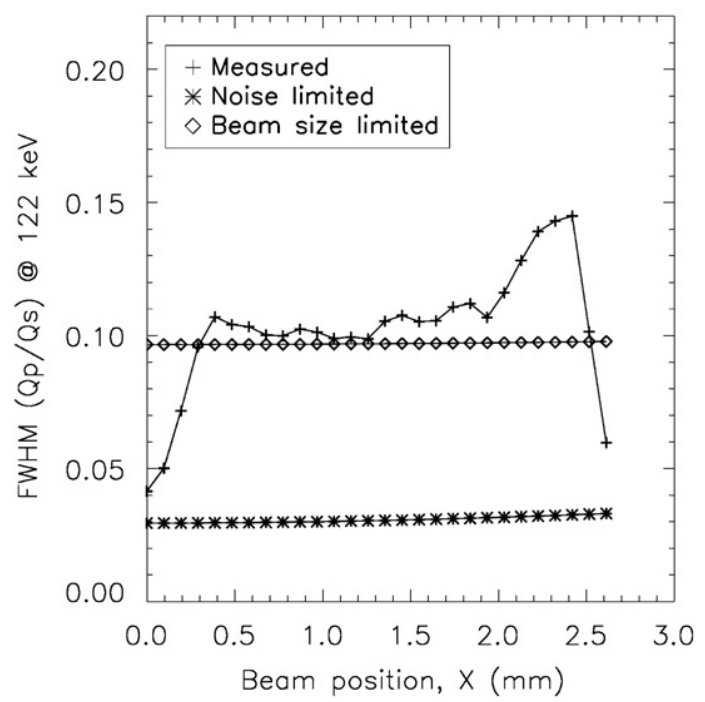

Fig. 7. Ratio resolution using depth sensing. (a) Measured and derived ratio as a function of beam position for 3 energies, (b) $R$ resolution shown for $122 \mathrm{keV}$. The minimum ratio resolution depends on non-correlated noise on detector signals $Q_{s}$ and $Q_{p}$. The finite beam size is the dominant noise source for the $R$ resolution.

that can be detected depends on the low energy threshold for $Q_{p}$ For the current detector scan setup the threshold was $6 \mathrm{keV}$ for both $Q_{p}$ and $Q_{s}$. Fig. 7(b) shows the measured and calculated depth resolution $R$ for the $122 \mathrm{keV}$ line. The depth resolution is calculated using Eq. (1) and shown with $*$. The finite beam size is the dominant contribution to the $R$ resolution and it is quadratically added to the electronic noise contribution, shown with $\diamond$. It is remarkable that the measured depth distribution for $x$-values close to zero is much narrower than the width of the beam. We believe that only a fraction of the beam hits the detector at the start positions and therefore is effectively more narrow. The minimum measured width is $100 \mu \mathrm{m}$ which is close to the depth resolution according to Eq. (1).

\section{Conclusion}

CZT drift strip detectors in PTF configuration are planned for the GRI high energy focal plane detector. A CZT drift strip detector was in this context evaluated in the PTF configuration for the first time: 2D scans were conducted illuminating a collimated ${ }^{57}$ Co photon beam $(230 \mu \mathrm{m} \varnothing)$ on a side of a $2.5 \mathrm{~mm}$ thick CZT drift strip detector. The detection efficiency was measured both in PPF ( $81 \%$ at $122 \mathrm{keV}$ ) and in PTF (99.8\% at $122 \mathrm{keV}$ ) configurations. The increased efficiency for the PTF configuration corresponds well with the larger effective thickness.

The energy resolutions were measured with photons from ${ }^{57} \mathrm{Co},{ }^{241} \mathrm{Am},{ }^{109} \mathrm{Cd}$ and ${ }^{137} \mathrm{Cs}$ sources in PPF and PTF. In both cases 
we achieved $3 \mathrm{keV}$ FWHM at $122 \mathrm{keV}$. The CTZ drift strip detector spectral performance for PTF and PPF are equal for the observed energy range.

CZT drift strip detectors in PTF configuration can achieve both high efficiency, due to the larger effective thickness, and good energy resolution, due to the small drift length for the electrons. They are therefore well suited for high energy astrophysics instrumentation.

The depth sensing technique of the drift strip detector was investigated performing 2D scans in the PTF configuration. The measured depth distributions were dominated by the beam size and their width was $300 \mu \mathrm{m}$ FWHM at $122 \mathrm{keV}$. The calculated minimum depth resolution is dependent on the electronic noise on the signals and it is $90 \mu \mathrm{m}$ FWHM at $122 \mathrm{keV}$ for the current detector setup. The present data indicate that this depth resolution is achievable. We plan to verify this at a facility where finer X-ray beams are available.

\section{References}

[1] M.A.J. van Pamelen, C. Budtz-Jørgensen, Nucl. Instr. and Meth. 403 (1998) 390.

[2] M.A.J. van Pamelen, C. Budtz-Jørgensen, Nucl. Instr. and Meth. 411 (1998) 197.

[3] E. Gatti, P. Rehak, in: Proceedings of 1983 DPF Workshop on Collider Detectors, Feb. 28-March 4, 1983, LBL-15973, UC-37, CONF-830224, p. 97.

[4] I. Kuvvetli, C. Budtz-Jørgensen, L. Gerward, C.M. Stahl, Radiat. Phys. Chem. 61 (2001) 457.

[5] I. Kuvvetli, Development of the CZT detector systems for space application, Ph.D. Thesis, ØrstedDTU, Technical University of Denmark, 2003. ISBN: 8791184-22-3.

[6] I. Kuvvetli, C. Budtz-Jørgensen, Nucl. Instr. and Meth. 52 (2005) 1975.

[7] C. Budtz-Jørgensen, I. Kuvvetli, N.J. Westergaard, P. Jonason, V. Reglero, C. Eyles, Nucl. Instr. and Meth. 458 (2001) 132.

[8] C. Budtz-Jørgensen, I. Kuvvetli, Y. Skogseide, K. Ullaland, N. Ostgaard, IEEE Trans. Nucl. Sci. NS-56 (4) (2008) 1842

[9] L. Natalucci, et al., Proc. SPIE 7011 (2008) 70111.

[10] E. Caroli, et al., Proc. SPIE 7011 (2008) 70113G. 